\title{
Classification of spoken words using surface local field potentials
}

\author{
Spencer Kellis, Kai Miller, Kyle Thomson, Richard Brown, Paul House, Bradley Greger
}

\begin{abstract}
Cortical surface potentials recorded by electrocorticography (ECoG) have enabled robust motor classification algorithms in large part because of the close proximity of the electrodes to the cortical surface. However, standard clinical ECoG electrodes are large in both diameter and spacing relative to the underlying cortical column architecture in which groups of neurons process similar types of stimuli. The potential for surface micro-electrodes closely spaced together to provide even higher fidelity in recording surface field potentials has been a topic of recent interest in the neural prosthetic community. This study describes the classification of spoken words from surface local field potentials (LFPs) recorded using grids of subdural, nonpenetrating high impedance micro-electrodes. Data recorded from these microECoG electrodes supported accurate and rapid classification. Furthermore, electrodes spaced millimeters apart demonstrated varying classification characteristics, suggesting that cortical surface LFPs may be recorded with high temporal and spatial resolution to enable even more robust algorithms for motor classification.
\end{abstract}

\section{INTRODUCTION}

CoRTICAL surface potentials recorded by in a variety of brain-machine interfaces (BMI) in recent years. Early studies demonstrated that data recorded from ECoG electrodes could be used to discriminate between motor and speech tasks and to discriminate phonemes [1, 2]. Subsequent work illustrated the viability of ECoG recordings in discriminating finger movement [3-5], arm movement [6, 7], and movement trajectories [8, 9].

An important advantage of ECoG electrodes is their close proximity to the sources of the recorded activity. Minimal separation between source and electrode allows ECoG recordings to provide relatively high signal-to-noise ratios and good spatial resolution. A question of significant recent interest is whether cortical surface potentials could be recorded in even higher spatial and temporal resolution than is possible using ECoG electrodes. Many researchers have

Manuscript received April 22, 2010. This work was supported in part by a Utah Research Foundation grant, DARPA RP2009 funding, NIH R01EY019363 (B.G.), and the Engineering Research Centers Program of the National Science Foundation under Award Number EEC-9986866 (R.B.).

B. Greger and K. Thomson are with the Department of Bioengineering, University of Utah, Salt Lake City, UT 84112, USA (email: bradley.greger@utah.edu).

S. Kellis and R. Brown are with the Department of Electrical and Computer Engineering, University of Utah, Salt Lake City, UT 84112, USA.

P. House is with the Department of Neurosurgery, University of Utah, Salt Lake City, UT 84112, USA. suggested that smaller electrodes and reduced inter-electrode spacing could improve the fidelity of the recorded data since correlated gamma activity has typically been limited to neighboring ECoG electrodes [1, 10]. These and similar observations are consistent with what might be expected intuitively from the underlying columnar organization of neurons processing similar types of information $[11,12]$. Given the scale of these cortical columns, usually in the hundreds of microns in diameter, a single $5 \mathrm{~mm}$ ECoG electrode would likely aggregate the activity of many such columns.

In this study, we used a recording device consisting of nonpenetrating microwire terminating at regular, millimeterscale intervals to record cortical surface potentials. The tight inter-electrode spacing of these micro-electrodes closely approximates the underlying columnar architecture of information processing in the cerebral cortex. We show that activity recorded by neighboring electrodes at this small scale supports classification of different words with high accuracy, and similarly, supports classification of the same word with different accuracies. With these results, this work extends previous studies in which similar micro-electrode arrays have been shown to support high temporal- and spatial-resolution recordings for BCI-like applications [6, $13]$.

\section{METHODS}

One male patient who required extraoperative ECoG monitoring for medically refractory epilepsy gave informed consent to participate in an institutional review boardapproved study. Two nonpenetrating micro-electrode arrays (PMT Neurosurgical, Chanhassen, MN) (fig. 1a), were placed beneath the dura mater over face motor cortex (FMC) and Wernicke's area (fig. 1b). The patient was instructed to articulate one of ten words (yes, no, hot, cold, hungry, thirsty, hello, goodbye, more, less) multiple times. For each word, a subset of 30 temporally proximal trials (15 for training and 15 for decode) containing stereotypical articulation was selected for further analysis. Trials were determined to be stereotyped by listening to them and judging each one individually.

Audio data from a microphone and 32 channels of neural data from two 16-channel micro-electrode arrays were recorded at 30,000 samples per second by a Neuroport system (Blackrock Microsystems, Salt Lake City, UT) during these experiments. Spectrograms of the neural data were generated using the open-source Chronux package software (chronux.org) with 400-msec windows and 100-msec step 

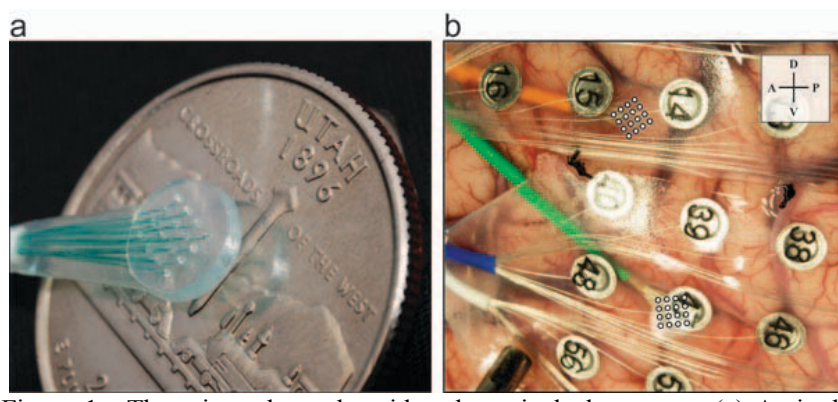

Figure 1. The micro-electrode grid and surgical placement. (a) A single 16-channel 4x4 micro-electrode grid shown next to a U.S. quarter-dollar coin. (b) photograph of micro-electrode grid surgical placement; the green wire bundle leads to the grid over Wernicke's area, and the orange wire bundle leads to the grid over face motor cortex.

size; tapering parameters were set to a time-bandwidth product of 5 and 9 leading tapers. Spectrograms were smoothed using a mean filter over four $0.9-\mathrm{Hz}$ bands and six 100 -msec time steps. Spectrograms were generated using data recorded when patient-researcher conversation was interspersed between the verbal tasks. Power between 70 and $200 \mathrm{~Hz}$ was calculated as a moving average of the squared magnitude of a bandpass-filtered signal.

Prior to classification, recorded data from each microelectrode were re-referenced by subtracting the common average of data from electrodes in the same array. For each spoken word, 0.5 seconds of data aligned to the articulation were extracted from all micro-electrodes and windowed by a Hann window. The power spectra of these data were calculated in 2-Hz bins between 0 and $500 \mathrm{~Hz}$ and lognormalized across trials for each micro-electrode. At the end of this process, each articulation was represented by 250 frequency-domain features.

We extended the method of Miller, et al. [14] to perform PCA on features from each micro-electrode and trial simultaneously. The input data matrix was constructed by stacking row vectors containing features from all electrodes for a specific trial. This matrix could be customized by including only features from a subset of micro-electrodes or trials. During the training phase, PCA was performed on a matrix consisting of features collected from 15 "training" trials per word. A center of mass, or centroid, was calculated for each word as the mean Euclidean coordinates of all relevant trials' feature-vectors projected into the principal component space. During the decode phase, projected feature-vectors from 15 additional trials were classified according to their proximity to one of the centroids.

The classification process was performed using features from combinations of two through ten words. Each incorporated word contributed 15 trials for training and 15 trials for decode. The term combination refers to the selection of $k$ unordered outcomes from $n$ possibilities, i.e., selecting $k$ of the available $n$ words where, in this case, $n=10$ and $k$ ranges between two and ten. For each set of outcomes, the mean, median, and standard deviation were computed for the accuracies of the classification results. Performance was

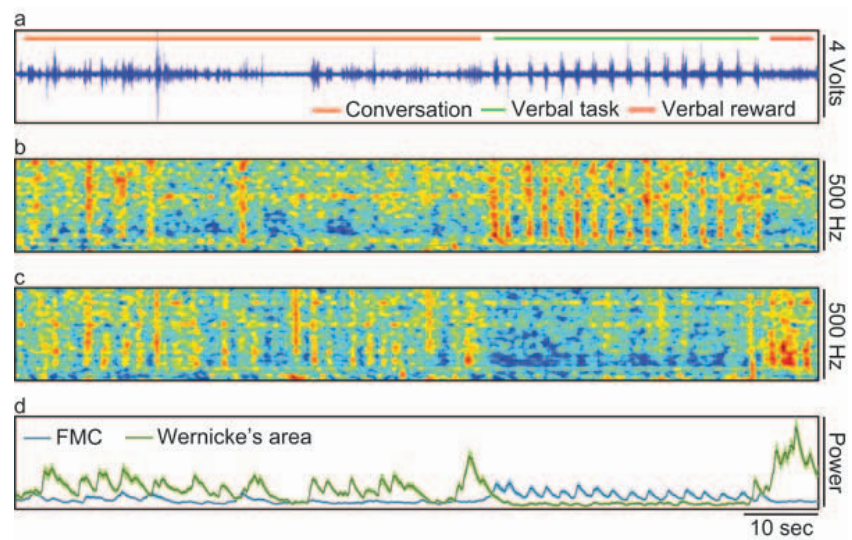

Figure 2. Raw data, spectrogram, and mean power during conversation and task. a, Audio waveform of conversation and verbal task in which the patient repeated the word "yes." b, Normalized spectrogram of neural data recorded from a single electrode over face motor cortex during the same time period shown in (a). c, Normalized spectrogram of neural data recorded from a single electrode over Wernicke's area during the same time period shown in (a). d, Mean power and standard error between 70 and 200 $\mathrm{Hz}$ for the 16 electrodes over FMC and the 16 electrodes over Wernicke's area.

also evaluated using features from one, five, 16, and 32 micro-electrodes at a time.

To assess the ability of individual micro-electrodes to discriminate individual words, features were selected from single micro-electrodes and evaluated once for each twoword combination. The accuracies of all classifications involving a given word were averaged to determine the ability of each micro-electrode to discriminate individual words against other possible class assignments.

Classification accuracy was measured against the level of chance for all evaluations. This level was determined by assuming equal likelihood of assignment to any class included in the training process (i.e., the uniform distribution applied to class assignment). For example, if two words were being classified, the level of chance was 0.5 since both potential class assignments were equally likely to be assigned to each trial. Classification accuracies consistently above the level of chance would indicate the detection and exploitation of relevant features from what could otherwise be completely stochastic physiological data.

\section{RESULTS}

Initial observations of neural signal recorded from face motor cortex revealed frequency-domain structure aligned to the individual words during the speech task (fig. 2). Conversely, Wernicke's area was predominantly active during conversation and while receiving verbal rewards after completing an experiment, and was less active during the repeated word experiments.

Data recorded from electrodes over face motor cortex were most classifiable, with $85.0 \pm 13.1 \%$ (mean \pm s.d.) of trials in two-word combinations correctly identified. Data recorded over Wernicke's area were less classifiable with $76.2 \pm 15.0 \%$ of trials in two-word combinations correctly 
classified. Using data recorded over both FMC and Wernicke's area jointly did not improve accuracy $(0.40 \pm$ $0.43 \%$ difference in the percentage of trials in two through ten-word combinations classified correctly).

Neighboring electrodes classified different words most accurately. For example, one electrode over FMC classified the word "no" with $89.3 \%$ average accuracy (average of all two-word classifications involving the word "no"), while a neighboring electrode $1 \mathrm{~mm}$ away classified the word "less" with $87.8 \%$ average accuracy. Of the 16 electrodes over FMC, 15 had a neighboring electrode within $1.4 \mathrm{~mm}$ (including diagonally situated neighbors) whose most accurately classified word, with at least $75 \%$ accuracy, was different. Fourteen of the 16 electrodes over Wernicke's area met the same criterion.

Conversely, neighboring electrodes classified the same word differently. For example, one electrode over FMC classified the word "hot" with $84.4 \%$ average accuracy while a neighboring electrode $1 \mathrm{~mm}$ away classified the same word with only $56.7 \%$ average accuracy (second column, top two electrodes). Eight out of 16 electrodes over FMC and five out of 16 electrodes over Wernicke's area classified their most accurate word at least 15 percentage points higher than a neighboring electrode classified the same word.

\section{DISCUSSION}

We have demonstrated that classification of articulated words from surface LFPs recorded on micro-ECoG grids can be performed both rapidly, i.e., within $500 \mathrm{msec}$ of the start of articulation, and with accuracy well above the level of chance. No patient training preceded the initial experimental session, so that classification was performed on features likely representing intuitive language processes. These results demonstrate the potential of using micro-electrodes designed to match the scale of cortical processing units, i.e., cortical columns, in BCI applications.

Due to the nature of the patient's medical history, it is possible that cortical language processing centers were relocated or otherwise altered. Furthermore, the repetitive nature of the patient experiments could mean that cognitive processes gave way to memory-based processes. While we confirmed as definitively as possible the parameters of this study regarding anatomy and language functioning, these potential issues could alter the conclusions of this research.

Studies of ECoG signals have shown that high gamma band modulation is correlated to motor actuation and occurs in more localized fashion, both temporally and spatially, than is evident in lower oscillatory bands [1, 15-18]. A common electrophysiological explanation for this behavior is that gamma oscillations represent the synchronizing (or synchronous) interactions of neuronal assemblies, perhaps cortical columns or macrocolumns, engaged in the parallel processing of common stimuli $[19,20]$. The coincidence of gamma-band event-related synchronization with motor tasks and the evidence for temporally discrete and topographically consistent modulation suggest this neural source is important to BCI operation. Recording these modulations at the appropriate scale requires grids of micro-electrodes matched to the underlying columnar scale.

Using micro-electrodes matched to the scale of cortical processing, we found variation of nearly $30 \%$ in the classification accuracies (for the same word) of individual electrodes spaced $1 \mathrm{~mm}$ apart. Within a $9-\mathrm{mm}^{2}$ space over FMC, almost every electrode had a neighbour within $1.4 \mathrm{~mm}$ whose most accurately classified word was not the same. This result suggests that in many cases electrodes recorded activity associated with features not as strongly represented in the data recorded by other nearby micro-electrodes. Recoding LFPs at the spatial scale of cortical columns appears to yield a broad set of relevant, discriminatory features that could serve as the underpinnings of an intuitive and rapid BCI for communication. While optimal parameters of spacing and electrode count are the subject of recent and ongoing studies [21], our findings motivate continued investigation into using micro-ECoG for BCI applications.

The results of the current study were obtained without any significant patient training. With some preparation, patients could adapt their neural processes to the performance of the classifier and learn to stereotype word articulation. More sophisticated classification algorithms could take advantage of stereotyped articulation to adapt to subtle differences in the cortical representations of different words. More sophisticated feature selection could improve performance as well.

The invasiveness of the micro-electrode grids could be further reduced with epidural placement, as has been shown for similar recording devices [21, 22]. Furthermore, a wireless implementation of the system might be practical given the relatively low bandwidth required to capture cortical surface LFPs. A wireless system able to record high-resolution cortical surface potentials could provide a reasonable balance of invasiveness and performance and improve the quality of life for locked-in patients and others unable to communicate on their own.

The tight inter-electrode spacing and small number of electrodes limited the spatial coverage of the micro-electrode grid. An optimized grid design with more electrodes would likely cover a larger number of relevant neural signals and allow better decoding accuracy. Optimal inter-electrode spacing and coverage is an important area for future research that will have wide implications in feature selection and classification algorithms.

\section{CONCLUSION}

We have presented a study in which spoken words were classified from cortical surface local field potentials. We demonstrated the capabilities of grids of nonpenetrating micro-electrodes to support robust algorithms for neural prosthetic applications. The accuracy of the classification 
process and the spatial variation observed at a millimeter scale warrant further investigation into optimal size and spacing of surface electrodes for brain machine interfaces.

\section{ACKNOWLEDGMENT}

The authors thank the EEG staff for assistance in conducting the study, and the patient who agreed to participate in the study.

\section{REFERENCES}

[1] E. C. Leuthardt, et al., "A brain-computer interface using electrocorticographic signals in humans.," J Neural Eng, vol. 1, pp. 63-71, 2004.

[2] T. Blakely, et al., "Localization and classification of phonemes using high spatial resolution electrocorticography (ECoG) grids," Conf Proc IEEE Eng Med Biol Soc, vol. 2008, pp. 49647, 2008.

[3] L. Bougrain and N. Liang, "Band-specific features improve Finger Flexion Prediction from ECoG," $J$ Argentinas Interfaces Cerebro Computadora, vol. 2009, pp. 1-4, 2009.

[4] R. Scherer, et al., "Classification of contralateral and ipsilateral finger movements for electrocorticographic brain-computer interfaces.," Neurosurg Focus, vol. 27, p. E12, 2009.

[5] S. Zanos, et al., "Electrocorticographic spectral changes associated with ipsilateral individual finger and whole hand movement," Conf Proc IEEE Eng Med Biol Soc, vol. 2008, pp. 5939-42, 2008.

[6] S. S. Kellis, et al., "Human neocortical electrical activity recorded on nonpenetrating microwire arrays: applicability for neuroprostheses.," Neurosurg Focus, vol. 27, p. E9, 2009.

[7] T. Pistohl, et al., "Prediction of arm movement trajectories from ECoG-recordings in humans.," J Neurosci Methods, vol. 167, pp. 105-14, 2008.

[8] G. Schalk, et al., "Decoding two-dimensional movement trajectories using electrocorticographic signals in humans," $J$ Neural Eng, vol. 4, pp. 264-75, Sep 2007.

[9] G. Schalk, et al., "Two-dimensional movement control using electrocorticographic signals in humans," J Neural Eng, vol. 5, pp. 75-84, Mar 2008.

[10] V. Menon, et al., "Spatio-temporal correlations in human gamma band electrocorticograms," Electroencephalog Clin Neurophysiol, vol. 98, pp. 89-102, 1996.

[11] V. B. Mountcastle, "Modality and topographic properties of single neurons of cat's somatic sensory cortex," $J$ Neurophysiol, vol. 20, pp. 408-34, Jul 1957.

[12] V. B. Mountcastle, "Brain mechanisms for directed attention," $J R$ Soc Med, vol. 71, pp. 14-28, Jan 1978.

[13] E. C. Leuthardt, et al., "Microscale recording from human motor cortex: implications for minimally invasive electrocorticographic brain-computer interfaces.," Neurosurg Focus, vol. 27, p. E10, 2009.

[14] K. J. Miller, et al., "Decoupling the cortical power spectrum reveals real-time representation of individual finger movements in humans.," J Neurosci, vol. 29, pp. 3132-7, 2009.

[15] N. Crone, et al., "Functional mapping of human sensorimotor cortex with electrocorticographic spectral analysis. II. Eventrelated synchronization in the gamma band," Brain, vol. 121, pp. 2301-2315, 1998.

[16] N. Crone, et al., "Functional mapping of human sensorimotor cortex with electrocorticographic spectral analysis. I. Alpha and beta event- related desynchronization," Brain, vol. 121, pp. 2271-2299, 1998.

[17] K. J. Miller, et al., "Spectral changes in cortical surface potentials during motor movement.," J Neurosci, vol. 27, pp. 2424-32, 2007.

[18] G. Pfurtscheller, et al., "Spatiotemporal patterns of beta desynchronization and gamma synchronization in corticographic data during self-paced movement.," Clini Neurophysiol, vol. 114, pp. 1226-36, 2003.

[19] N. E. Crone, et al., Event-Related Dynamics of Brain Oscillations vol. 159: Elsevier, 2006.

[20] W. Singer, "Synchronization of cortical activity and its putative role in information processing and learning," Annu Rev Physiol, vol. 55, pp. 349-74, 1993.

[21] M. W. Slutzky, et al., "Optimal spacing of surface electrode arrays for brain-machine interface applications," J Neural Eng, vol. 7, p. 26004, Apr 2010.

[22] E. C. Leuthardt, et al., "Electrocorticography-based brain computer interface--the Seattle experience," IEEE Trans Neural Syst Rehabil Eng, vol. 14, pp. 194-8, Jun 2006. 